\title{
Hallazgos en endosonografía anorrectal en una cohorte prospectiva
}

\section{Findings from anorectal endosonography in a prospective cohort}

\author{
Edgar Julián Ferreira B., MD, ${ }^{1}$ Lázaro Antonio Arango M., MD, ${ }^{2}$ Edelberto Mulett V., MD, ${ }^{3}$ Mauricio Osorio Ch., MD, ${ }^{3}$
}

Dínimo José Bolívar S., MD. ${ }^{1}$

\footnotetext{
1 Fellow Gastroenterología Clínico-Quirúrgica, Universidad de Caldas. Manizales, Colombia.

2 Coordinador Programa de Gastroenterología

Clínico-Quirúrgica, Universidad de Caldas. Docente Gastroenterologia Clínico-Quirúrgica,

Universidad de Caldas. Manizales, Colombia.

3 Docente Gastroenterologia Clínico-Quirúrgica,

Universidad de Caldas. Manizales, Colombia.

Fecha recibido: $\quad 02-12-15$

Fecha aceptado: 26-01-16
}

\begin{abstract}
Resumen
La endosonografía anorrectal permite diagnosticar múltiples patologías del canal anal y del recto. En Colombia se realiza en pocos sitios y es escaso el personal entrenado para ello. Un grupo de docentes de gastroenterología clínica quirúrgica de la Universidad de Caldas recibió entrenamiento en endosonografía anorrectal en Chile y España, después de lo cual inició su trayectoria, ya de 4 años. Con los datos del último año se realizó un estudio descriptivo en una cohorte prospectiva, tomando la información de los pacientes a quienes se les realizó endosonografía anorrectal entre octubre de 2014 y septiembre de 2015. Se obtuvieron datos de 136 pacientes, predominando el género femenino (72,05\%); el 20,6\% de los pacientes procedían de departamentos diferentes a Caldas. La principal indicación fue incontinencia fecal en 44 pacientes (32,35\%), siendo $86 \%$ mujeres; otras indicaciones fueron neoplasia maligna rectal $(23,5 \%)$, fístula perianal $(12,5 \%)$, proctalgia $(11,03 \%)$, endometriosis $(7,35 \%)$ y absceso perianal $(6,62 \%)$. El diagnóstico final más frecuente fue defecto de los esfínteres en 40 pacientes $(29,41 \%)$, seguido por neoplasia maligna rectal (13,24\%), fístula perianal $(11,76 \%)$, absceso perianal $(8,82 \%)$ y neoplasia benigna rectal $(6,62 \%)$. Los resultados están en congruencia con la literatura mundial, donde la principal indicación es la incontinencia fecal en relación con el diagnóstico final, que es defectos de los esfínteres por alteraciones obstétricas.

La endosonografía anorrectal es de gran importancia como método diagnóstico único o de apoyo para otros, con centros de referencia como el nuestro, donde se realiza en un volumen importante y siguiendo las pautas internacionales recomendadas.
\end{abstract}

\section{Palabras clave}

Endosonografía, canal anal, recto, incontinencia fecal

\section{INTRODUCCIÓN}

La endosonografía en el tracto gastrointestinal empezó a utilizarse desde 1993, y los avances más vertiginosos en el área anorrectal se han dado en la última década, logrando igualar y en ocasiones superar el rendimiento de otros estudios establecidos previamente para tal fin (1). En Chile existe una publicación de 1000 casos de pacientes a los que se les realizó endosonografía anorrectal, describiendo sus principales indicaciones y diagnósticos endosonográficos finales; esta corresponde a una de las series con mayor número de pacientes reportados en quienes se haya realizado este examen (4). En Colombia existen muy pocos estudios sobre la utilización de la endosonografía en el tracto gastrointestinal, con muy poca información sobre los centros de referencia donde se realiza este procedimiento $(2,3)$.

En la actualidad, las indicaciones más frecuentes para la realización de este examen son el estudio de incontinencia fecal, fístulas perianales, absceso perianal, estadificación y seguimiento de cáncer de ano o recto, y dolor rectal (4).

El rendimiento diagnóstico de la endosonografía anorrectal varía dependiendo de la indicación del examen, con 


\begin{abstract}
Anorectal endosonography can diagnose many diseases of the anal canal and rectum. In Colombia, the procedure is performed in only a few medical centers because of the scarcity of trained personnel. Four years ago, a group of teachers from the gastroenterological surgery clinical at the University of Caldas received training in endoluminal ultrasonography in Chile and Spain and have been practicing these procedures ever since. This is a descriptive study of a prospective cohort using data from patients who underwent endoluminal ultrasonography between October 2014 and September 2015. Data were obtained from 136 patients, of whom 72.05\% were women. $20.6 \%$ of the patients came from departments other than Caldas. Fecal incontinence was the reason for the procedure for 44 patients $(32.35 \%, 86 \%$ of whom were women). Other indications were rectal malignancies $(23.5 \%)$, perianal fistulas $(12.5 \%)$, anal pain $(11.03 \%)$, endometriosis $(7.35 \%)$, and perianal abscesses (6.62\%). The most common final diagnoses were defective sphincters in 40 patients $(29.41 \%)$, rectal malignancy in $13.24 \%$ of the patients, perianal fistulas in $11.76 \%$ of the patients, perianal abscesses in $8.82 \%$ of the patients, and benign rectal neoplasms in $6.62 \%$ of the patients. The results are consistent with those found in the international literature where the most common final diagnoses reported for the reason for fecal incontinence are sphincter defects and obstetric disorders.

Anorectal endosonography is of great importance when used alone or to support other diagnostic tools. It is recommended that this procedure be done in a referral center such as ours which performs a large volume of these procedures and which follows international guidelines.
\end{abstract}

\title{
Keywords
}

Endosonography, anal canal, rectum, fecal incontinence.

una sensibilidad cercana al 100\% para abscesos perianales, de $87 \%$ para fístulas perianales, entre $82 \%-95 \%$ para tumores y menor para otras indicaciones (5-7).

Un grupo de docentes de gastroenterología clínica quirúrgica de la Universidad de Caldas recibió entrenamiento en la realización de endosonografía anorrectal en Chile y luego en España, después de lo cual se inició una experiencia propia que ya tiene 4 años y resultados muy satisfactorios.

Con la intención de dar a conocer la experiencia sobre la utilización de endosonografía anorrectal, se propuso como objetivo describir los hallazgos de esta en una cohorte prospectiva de pacientes adultos atendidos en Unión de cirujanos, Manizales, entre octubre de 2014 y septiembre de 2015.

\section{MATERIALES Y MÉTODOS}

Se realizó un estudio descriptivo en pacientes adultos y de ambos sexos a los que se les practicó endosonografía anorrectal (por cualquier indicación) por el grupo de gastroenterología clínica quirúrgica de la Universidad de Caldas, en Unión de cirujanos, clínica La Presentación, entre octubre de 2014 y septiembre de 2015 . El criterio de exclusión fue pacientes en quienes no se pudiera recolectar la totalidad de los datos.

Se utilizó un instrumento de recolección de datos previamente diseñado, en el que se tomó información personal (nombre, edad, sexo, ocupación), demográfica (lugar de procedencia), tiempo de evolución de la enfermedad actual relacionada con la indicación del examen, indicación del estudio, diagnóstico endosonográfico final y los hallazgos de medición de las estructuras anatómicas en el canal anal; además, en mujeres se preguntó la historia ginecológica y/o de desgarros durante sus partos.

\section{Técnica del procedimiento}

Para todos los exámenes se utilizó un endosonógrafo radial estándar con visión endoscópica y endosonográfica en el mismo equipo. Se utilizaron frecuencias entre 5 y $15 \mathrm{MHz}$, según la necesidad. El procesador de la unidad utilizado es de alta resolución.

Los pacientes fueron evaluados en posición de decúbito lateral izquierdo, luego de esto se realizó inspección perianal detallada, tacto rectal y evaluación endoscópica hasta el recto superior; se procedió a la evaluación endosonográfica localizando como estructura anatómica guía en nivel anterior el útero (o vejiga cuando el útero estaba ausente) en la mujer, o la próstata (uretra masculina cuando la próstata estaba ausente) en el hombre, además de la localización del músculo puborrectal en nivel posterior para ambos. La izquierda fue localizada a las 3 del reloj y la derecha a las 9 del reloj. En caso de evaluación de lesiones tipo masa, se utilizó agua como medio acústico; asimismo, en casos donde se estudió fístula perianal, se insertó una cánula en el orificio secundario perianal con la porción no punzante de un catéter 18 o $20 \mathrm{G}$, se inyectó agua oxigenada para generar un efecto de contraste a través del trayecto fistuloso y para que fuera visible endosonográficamente en toda su extensión. Se realizó medición del grosor de los esfínteres a las 3 o 9 del reloj, medición de las masas, y se aplicó el sistema 
internacional TNM de estadificación tumoral actualizado para tumores de recto y ano. Para la medición del cuerpo perineal femenino, se realizó tacto vaginal simultáneo y se tomó la medida desde la visión del dedo enguantado visto hiperecogénico hasta la mucosa.

\section{Análisis de datos}

Los datos descriptivos están expresados como porcentajes, medias y desviación estándar.

\section{RESULTADOS}

Se realizaron 162 endosonografías anorrectales, sin lograr tomar los datos de 26 pacientes por diferentes motivos, por lo que se lograron obtener los datos de 136 pacientes.

\section{Características demográficas}

Hubo un predominio de sexo femenino $72,05 \%(\mathrm{n}=98)$. El promedio de edad fue similar para el grupo masculino y femenino: 55,6 (DE 14,46) y 53,5 años (DE 14,98), respectivamente. El 79,4\% de los pacientes procedía del departamento de Caldas, mientras que porcentaje restante procedía de Risaralda, Valle, Quindío o Antioquia (tabla 1).

Tabla 1. Datos demográficos.

\begin{tabular}{lc}
\hline \multicolumn{1}{c}{ Variable } & Valor \\
\hline Edad (años) promedio (máxima-mínima) en mujeres & $53,5(23-87)$ \\
Edad (años) promedio (máxima-mínima) en hombres & $55,6(23-83)$ \\
& $\%$ (Frecuencia) \\
Sexo femenino & $72,05 \%(98)$ \\
Procedencia & \\
Caldas & $79,4 \%(108)$ \\
Risaralda & $10,29 \%(14)$ \\
Valle & $5,15 \%(7)$ \\
Quindío & $2,94 \%(4)$ \\
Antioquia & $2,21 \%(3)$ \\
\hline
\end{tabular}

\section{HISTORIA GINECOLÓGICA}

De las 98 pacientes de sexo femenino, 34 reportaron historia de partos vaginales, y de estas, 30 reportaron historia de desgarro durante alguno de sus partos $(88,2 \%)$; el número máximo de partos reportados fue de 6 y el mínimo de 1 .

\section{Indicación del examen}

Por estudio de incontinencia fecal se encontraron 44 pacientes (32,35\%); la mayoría fueron mujeres $(86 \%, \mathrm{n}$
=38) (tabla 2 y figura 1). Las indicaciones de fístula perianal y absceso perianal tuvieron una frecuencia de $12,5 \%$ y $6,62 \%$, respectivamente. Dentro del grupo de incontinencia, 28 de las 38 mujeres que la reportaron también tenían historia de desgarro obstétrico $(76,7 \%)$. El total de las indicaciones está resumido en la tabla 3.

Tabla 2. Estudio por incontinencia.

\begin{tabular}{cc}
\hline \multicolumn{2}{c}{ Indicación del examen - incontinencia } \\
\hline Género & Incontinencia \\
\hline Femenino & 38 \\
Masculino & 6 \\
\hline
\end{tabular}

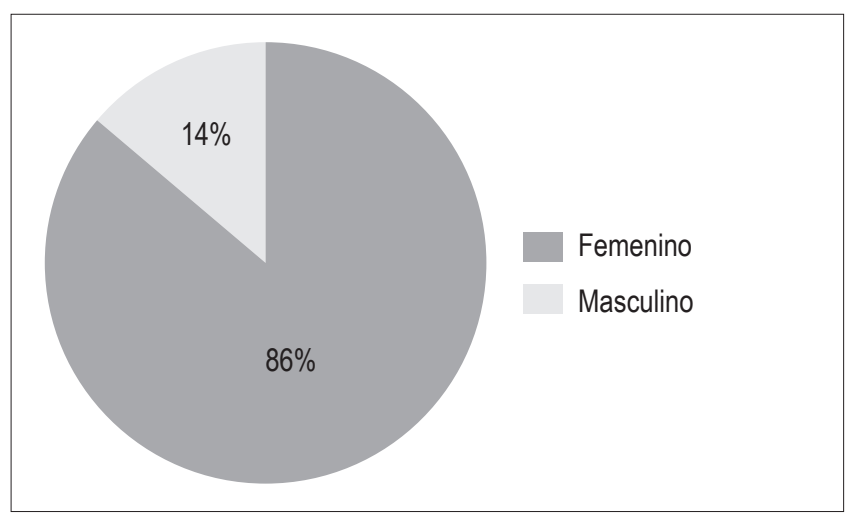

Figura 1. Gráfica del estudio por incontinencia.

Tabla 3. Listado de indicaciones endosonografía anorrectal.

\begin{tabular}{lc}
\hline \multicolumn{1}{c}{ Indicación del examen } & $\begin{array}{c}\text { Frecuencia } \\
\text { (Número de casos) }\end{array}$ \\
\hline Incontinencia & $32,35 \%(44)$ \\
Fístula perianal & $12,5 \%(17)$ \\
Neoplasia maligna rectal confirmada & $12,5 \%(17)$ \\
Neoplasia maligna rectal sin confirmar & $11,03 \%(15)$ \\
Proctalgia & $11,03 \%(15)$ \\
Endometriosis & $7,35 \%(10)$ \\
Absceso perianal & $6,62 \%(9)$ \\
Neoplasia maligna anal confirmada & $3,68 \%(5)$ \\
Neoplasia benigna rectal & $2,94 \%(4)$ \\
Obstrucción al tracto de salida & $2,21 \%(3)$ \\
Neoplasia maligna anal sin confirmar & $0,74 \%(1)$ \\
Neoplasia benigna anal & $0,74 \%(1)$ \\
Úlcera solitaria de recto & $0,74 \%(1)$ \\
\hline
\end{tabular}

\section{Diagnóstico endosonográfico final}

Se encontró defecto de los esfínteres en 40 pacientes $(29,41 \%)$, de los cuales 6 fueron hombres y 34 mujeres; 
aquí cabe anotar que el 93,33\% ( $\mathrm{n}=28)$ de las mujeres que tienen historia de incontinencia, también tienen antecedente de desgarro. De las 34 mujeres con defectos en los esfínteres, 32 lo tuvieron localizado en canal anal medio, con compromiso en nivel anterior, 1 de ellas con atrofia difusa de esfínteres, y otra con compromiso de canal anal inferior (figura 2).

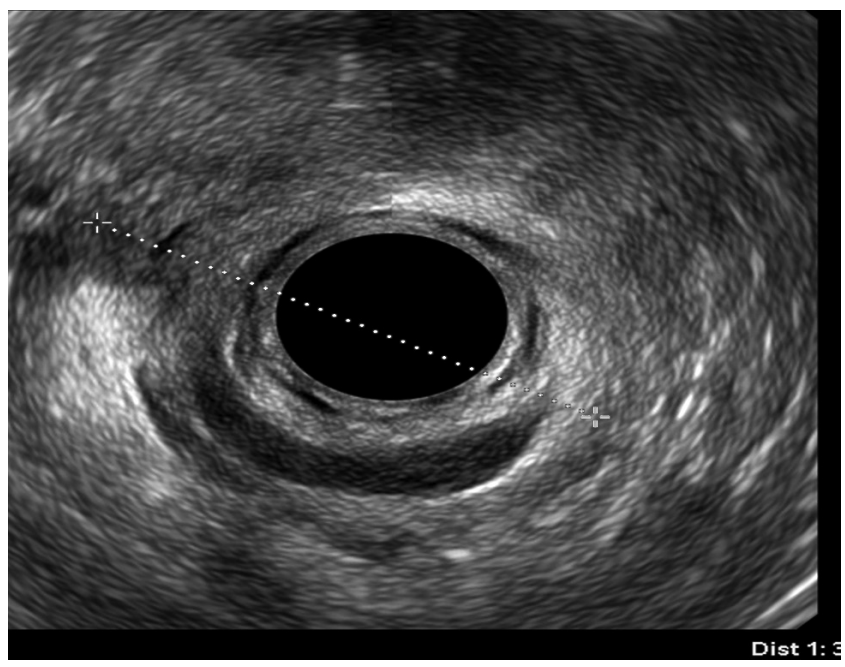

Figura 2. Canal anal medio con defecto y ausencia de esfínteres en media circunferencia anterior, $180^{\circ}$.

Por otro lado, en el grupo masculino con estudio por incontinencia, el diagnóstico en todos fue cambios por atrofia del aparato esfinteriano, que pudo verse en cualquiera de las localizaciones del canal anal y que se evidenció como engrosamiento de los músculos en general y cambios difusos de la ecogenicidad de los mismos, sin posibilidad de determinar el borde que los delimita. Se encontró fístula perianal en 16 pacientes $(11,8 \%)$; la principal forma de presentación de las fístulas fue transesfintérica $(37,5 \%)$, seguida por las interesfintéricas y anovaginales (figuras 3 y 4 ). Doce pacientes presentaron absceso perianal ( $8,8 \%)$; de estos, el $83 \%$ correspondió a abscesos submucosos o isquiorrectales (figura 5).

Los demás diagnósticos finales se presentan en la tabla 4. Es de resaltar que se pudo determinar en el grupo diagnosticado como neoplasia benigna de recto, la posibilidad de resección endoscópica en lesiones que tenían planeadas cirugías más invasivas (figura 6 y 7), así como la posibilidad de determinar la recuperación del aparato esfinteriano en 3 pacientes en seguimiento por cáncer de canal anal después de manejo por parte de oncología. En 2 pacientes de los diagnosticados con neoplasia maligna de recto se identificó lesión menor a T2 y N0 según las clasificación de TNM (figura 8), considerándose lesiones tempranas; en el otro $88,8 \%$ se encontraron lesiones avanzadas (figura 9 y 10 ).
También se anota que, dentro de la categoría otros, están con orden de frecuencia de mayor a menor endometriosis o endometrioma, enterocele, fisura anal, várices rectales y un caso de cáncer de colon sigmoides.

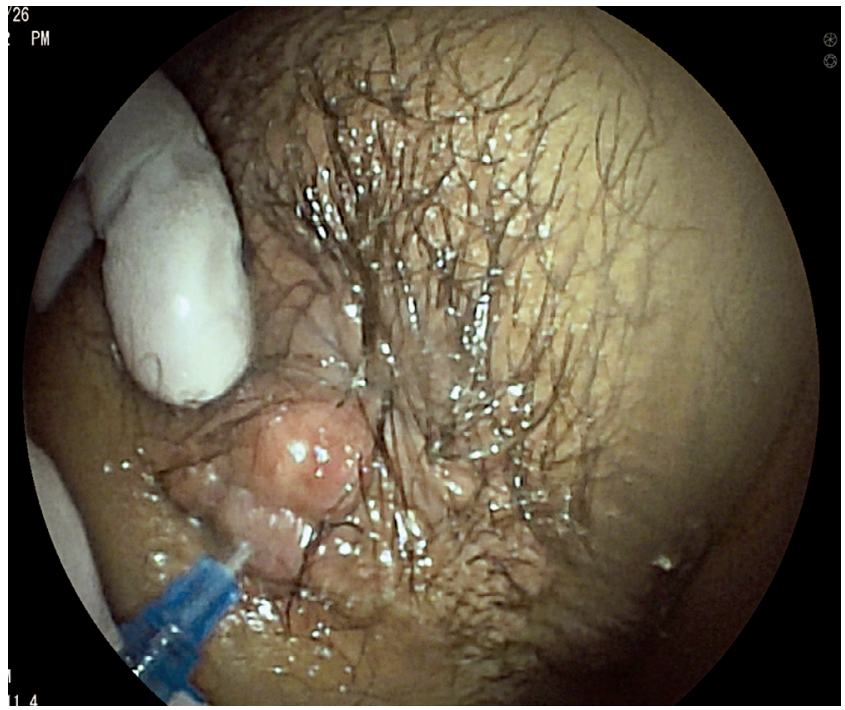

Figura 3. Orificio secundario de fístula perianal canalizado con intracat para inyección de agua oxigenada.

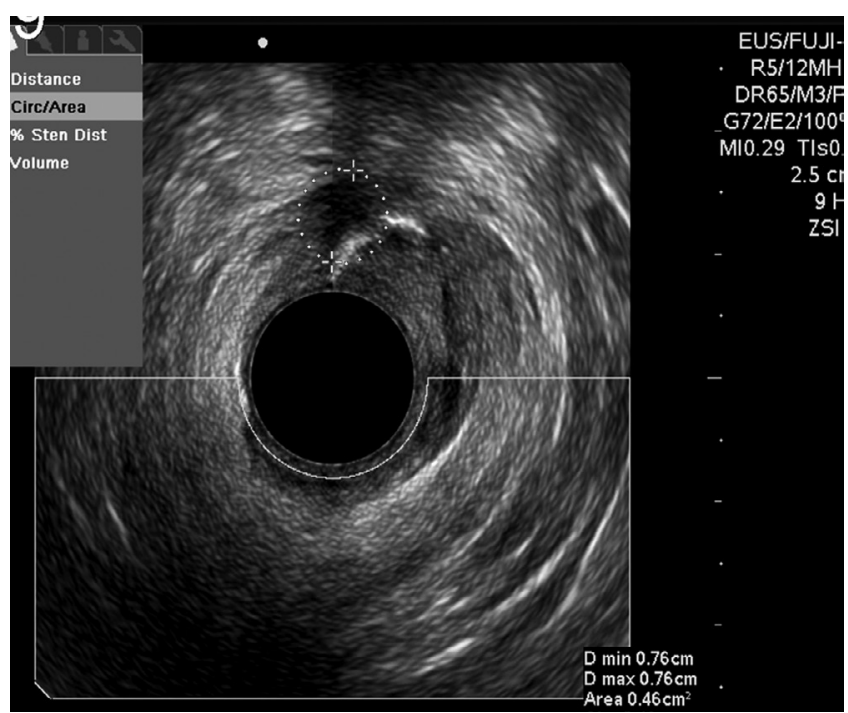

Figura 4. Fístula transesfintérica entre canal anal medio e inferior, dibujando trayecto con inyección de agua oxigenada.

Dentro del resultado normal, las indicaciones de mayor a menor frecuencia fueron estudio de proctalgia $(n=5)$, neoplasia de recto no confirmada $(\mathrm{n}=2)$, endometriosis ( $\mathrm{n}$ $=2)$, incontinencia fecal $(\mathrm{n}=2)$, neoplasia anal no confirmada ( $\mathrm{n}=2)$, y un caso fístula perianal, absceso perianal, úlcera solitaria de recto y obstrucción al tracto de salida. 


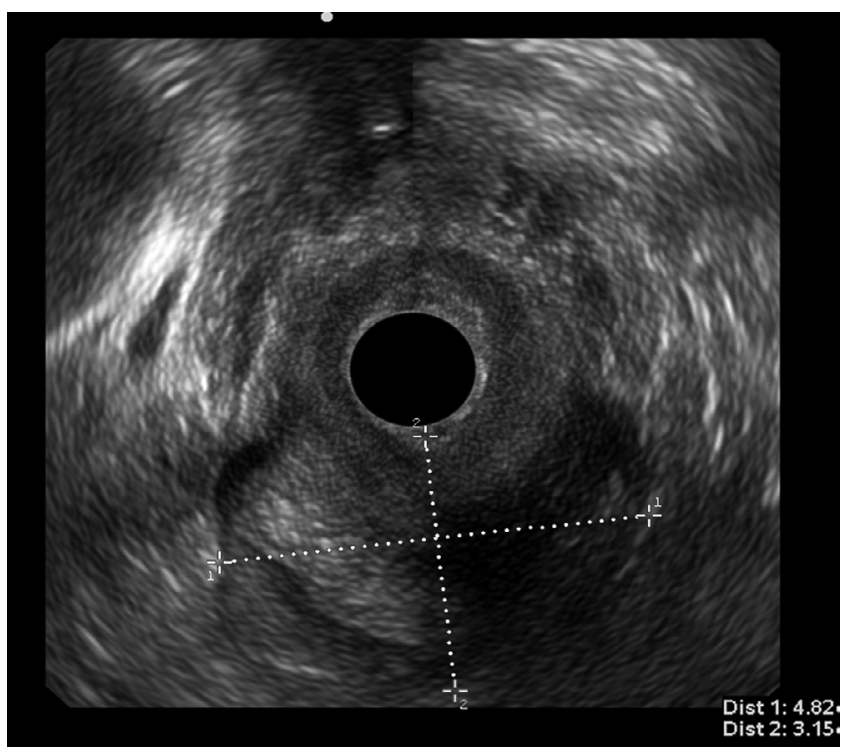

Figura 5. Gran absceso isquiorrectal con componente en herradura.

Tabla 4. Diagnóstico endosonográfico final.

\begin{tabular}{lc}
\hline \multicolumn{1}{c}{ Diagnóstico final } & Frecuencia (número de pacientes) \\
\hline Defecto de esfínteres & $29,41 \%(40)$ \\
Neoplasia maligna rectal & $13,24 \%(18)$ \\
Fístula perianal & $11,76 \%(16)$ \\
Absceso perianal & $8,82 \%(12)$ \\
Neoplasia benigna de recto & $6,62 \%(9)$ \\
Neoplasia maligna anal & $1,47 \%(2)$ \\
Normal & $11,76 \%(16)$ \\
Otro & $16,91 \%(23)$ \\
\hline
\end{tabular}

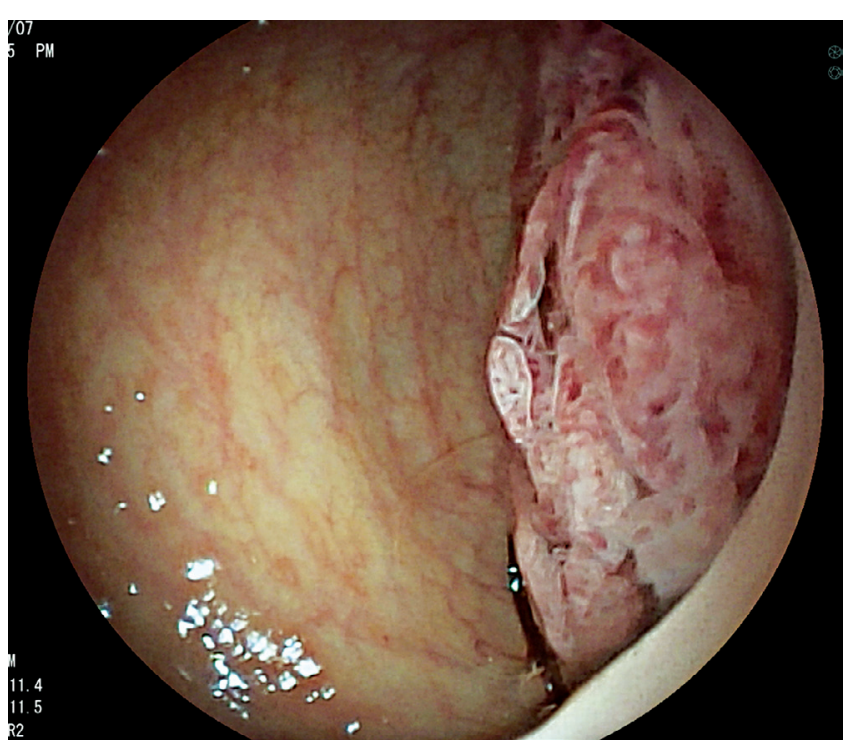

Figura 6. Adenoma velloso de recto inferior.

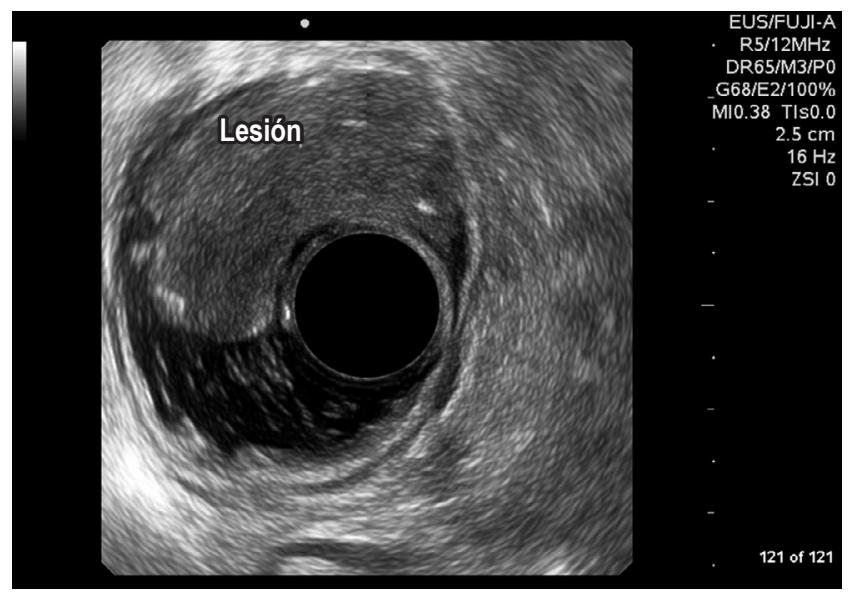

Figura 7. Adenoma velloso de recto inferior, compromiso hasta capa III (submucosa) y posibilidad de resección endoscópica.

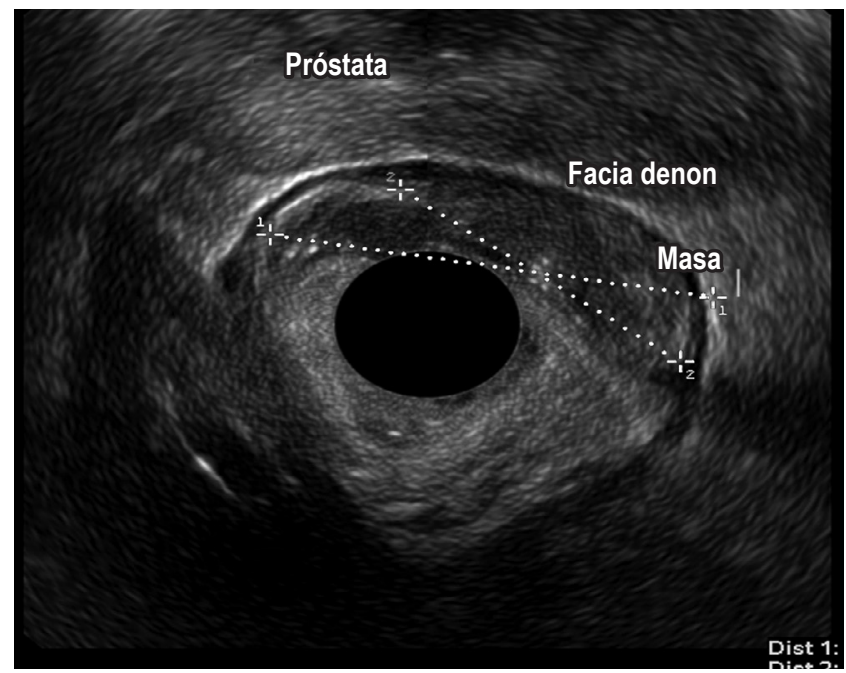

Figura 8. Tumor maligno de recto inferior que compromete la musculatura propia sin sobrepasar $\mathrm{T} 2$.

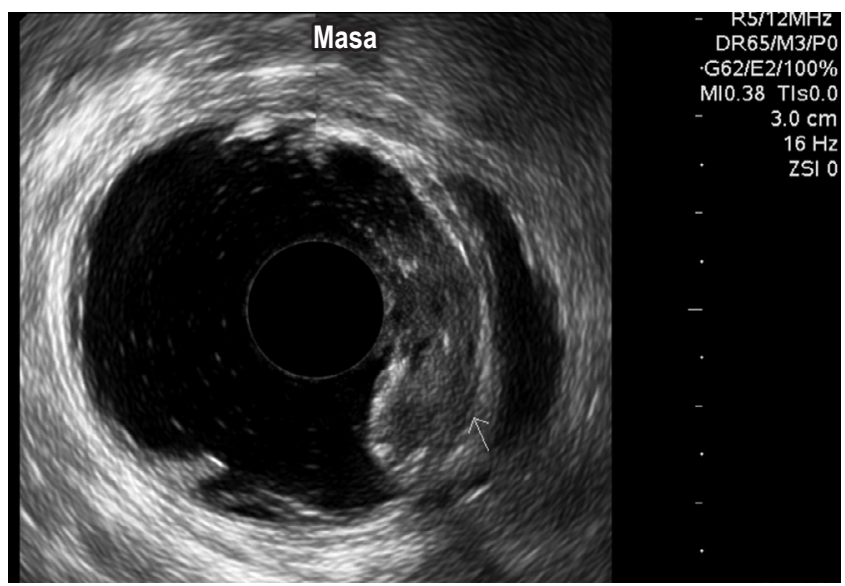

Figura 9. Tumor de recto que se sale de la capa V endosonográfica y compromete tejido perirrectal. 


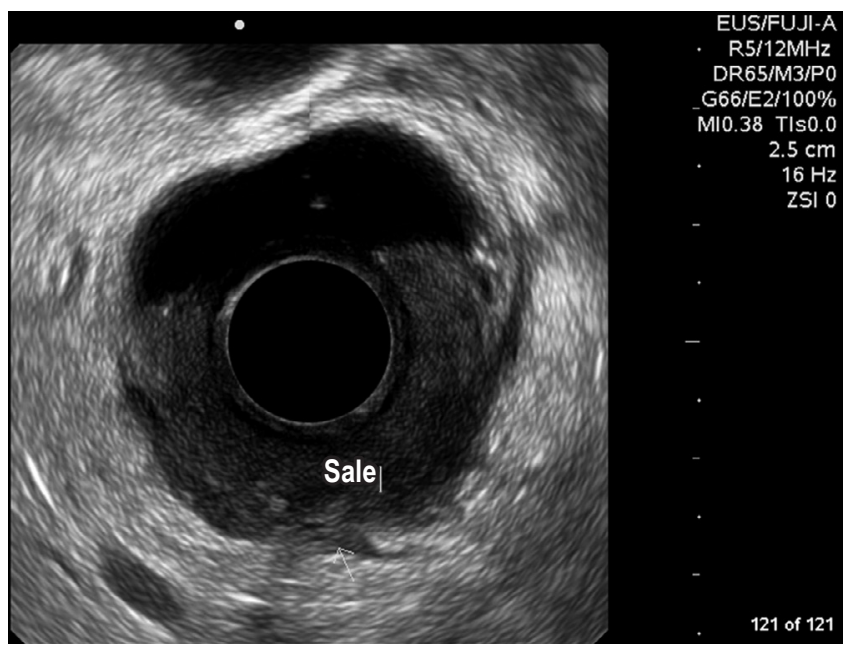

Figura 10. Tumor maligno de recto que se sale de la capa V endosonográfica, compromete tejido perirrectal y se asocia con adenopatía de aspecto tumoral maligno periférica.

\section{Medidas de los esfínteres}

La medida del puborrectal fue reportada como normal en el $92,59 \%$ de los pacientes $(n=125)$. En el canal anal superior, la medida del esfínter anal interno fue reportada normal en todos los casos. En el canal anal medio, todas las medidas de esfínter anal interno son reportadas como normales. Las medidas del esfínter anal externo en el canal anal medio son reportadas como normales en el $97,78 \%$ de los casos $(\mathrm{n}=132)$. Todas las medidas del esfínter anal externo en el canal anal inferior fueron reportadas como normales. La medida del cuerpo perineal fue reportada como anormal en el 11,22\% de las mujeres ( 11 de las 98 ).

\section{DISCUSIÓN}

La principal indicación de la endosonografía fue incontinencia fecal, con una mayoría de población femenina, lo cual está en relación con la literatura mundial (12-16); también es congruente que en las mujeres con indicación del estudio por incontinencia predominó la historia de desgarro obstétrico. En su estudio descriptivo, López-Kôstner y colaboradores (4) obtuvieron como principal indicación de endosonografía anorrectal, la incontinencia fecal, presentándose en 336 pacientes de 1000 (33,6\%); muy en relación con nuestro reporte, donde fue de $44 \%$. De igual forma, dicha indicación fue predominante en mujeres (86\%), idéntico a nuestros datos.

La presencia de fístulas perianales como segunda indicación en frecuencia realza la importancia de este estudio, donde se puede caracterizar con precisión la anatomía quirúrgica para lograr una clasificación adecuada de la fístula.
Diversos autores también utilizan el agua oxigenada como elemento de contraste en la visión endosonográfica para así poder detallar el trayecto fistuloso $(4,8,17,18)$.

De igual forma, la importante frecuencia de indicación en pacientes con neoplasia maligna rectal confirmada habla de la aceptación a nivel nacional y regional que se le está dando a este diagnóstico por parte de grupos interdisciplinarios de oncología, donde la endosonografía juega un papel fundamental a la hora de complementar otras ayudas diagnósticas para estadificación locorregional y planeamiento adecuado de la terapia $(4,9,19-22)$.

El estudio de la proctalgia, siendo tan complejo por la fisiopatología incomprendida del todo y por la alta posibilidad de no encontrar hallazgos imagenológicos o endosonográficos, también ha logrado espacio con la endosonografía anorrectal (10).

Al considerar el defecto esfinteriano como principal diagnóstico final se refuerza la principal indicación, incontinencia fecal, y van de la mano al final, como también lo hace la mayoría femenina con historia de desgarros obstétricos dentro de este diagnóstico $(10,11)$.

Existe un grupo importante de pacientes cuya endosonografía fue reportada como normal con indicaciones diversas, donde se destaca el diagnóstico mayoritario de proctalgia; incluso dentro del grupo al que se le indicó este estudio por proctalgia, el 33\% reportó un examen normal, lo cual está en congruencia con lo reportado en la literatura mundial (23-26).

Las medidas de los esfínteres están reportadas como normales en la gran mayoría de los casos, pero esto está influenciado porque dichas medidas se realizan a las "9" y "3" del reloj (aspecto lateral de los esfínteres), lo cual dista de la presencia de las alteraciones encontradas en las endosonografías por incontinencia, por ejemplo, que son en nivel anterior, y esta es la indicación más frecuente (24-27).

La medida del cuerpo perineal estuvo disminuida en un número significativo de mujeres, en relación también con la incontinencia como principal indicación.

El presente trabajo resalta que la realización del estudio endosonográfico en nivel anorrectal se viene practicando ya en el país en instituciones como la nuestra, siguiendo los estándares internacionales y con las indicaciones que dicta la norma. También es evidente que existe un volumen de trabajo importante, convirtiendo al grupo de gastroenterología clínica quirúrgica de la Universidad de Caldas en centro de referencia en el país para este tipo de examen.

En el presente estudio llama la atención que, a pesar de tener una clara mayoría de pacientes originarios del propio departamento de Caldas, un porcentaje superior al $20 \%$ procedía de otros lugares; lo anterior resalta el hecho de que este grupo de referencia recibe pacientes de varias partes del país. 
También realza las dificultades para el acceso a la salud que se viven en Colombia, donde se requieren muchas veces grandes desplazamientos para obtener un servicio.

Se trata de un estudio sencillo, punto de partida para estimular la realización de otros, con tecnología que cada vez toma más fuerza a nivel mundial y requiere estar a la vanguardia de los adelantos que lleguen. Permite la posibilidad de tener en cuenta las principales indicaciones en nuestro medio para la endosonografía anorrectal, a partir de lo cual las instituciones prestadoras de salud pueden desarrollar guías de práctica clínica donde se tengan en cuenta esas indicaciones, logrando así beneficios enormes para los pacientes.

\section{REFERENCIAS}

1. Armengol JR, Benjamin S, Binmoeller K. Consensus Conference. Clinical applications of endoscopic ultrasonography in gastroenterology: state of the art. Endoscopy. 1993;25:358-66.

2. GómezM, Otero W, Arbeláez V, RodríguezJ.Ecoendoscopia. Indicaciones de la A a la Z. Rev Col Gastroenterol. 2005;20(2):34-64.

3. De Nubbila E, Montes J, Spath A, Serrano S, Díaz-Yúñez I, Parra G. Endosonografía del esfínter rectal. Rev Colomb Radiol. 2007;18(4):2233-9.

4. López-Köstner F, Zárate A, García-Huidobro M, Pinedo G, Molina M, Krônberg $\mathrm{O}$, et al. Indicaciones y resultados de la endosonografía anorrectal. Análisis de las primeras 1000 endosonografías. Rev Chil Cirug 2007;50(1):31-37.

5. Alós R, de la Portilla, F. La ecografía anorrectal en las neoplasias de recto. En: Principios Prácticos de Ecografía anal y Rectal. Madrid. 2004. Ed. Díaz de Santos. 81- 95.

6. Garcés M, García S, Esclápez P, Sanahuja A, Espí A, Flor B, et al. Evaluación de las fístulas perianales mediante ecografía endoanal tridimensional y correlación con los hallazgos intraoperatorios. Cir. Esp. 2010;87(5):299-305.

7. Stamatiadis A. Endoanal ultrasound in perianal abscesses. Dis Colon Rectum. 2002;45:710-11.

8. Glaser F, Schlag P, Herfarth C. Endorectal ultrasonography for the assessment of invasion of rectal tumours and lymph node involvement. Br J Surg. 1990;77:883-887.

9. Jordán J, Roig JV, García Armengol J, Esclápez P, Jordán Y, García Granero E, Alós R, Lledó S. Importancia de la exploración física y técnicas de imagen en la valoración diagnóstica de las fístulas de ano. Cir.Esp. 2009;85(4):238-245.

10. Alos R, Roig JV. Utilidad y limitaciones de la ecografía anorrectal. Rev Sdad Valenciana Patol Dig. 1999;18:56-67.

11. Saranovic D, Barisic G, Krivokapic A, Masulovic D, DjuricStefanovic A. Endoanal ultrasound evaluation of anorectal diseases and disorders: Technique, indications, results and limitations. Eur J Radiol. 2007;61:480-489.

12. Starck M, Bohe M, Valentin L. The extent of endosonographic anal sphincter defects after primary repair of obstetric sphincter tears increases over time and is related to anal incontinence. Ultrasound Obstet Gynecol. 2006;27(2):188-97.

13. Oberwalder M, Thaler K, Baig MK, Dinnewitzer A, Efron J, Weiss EG, Vernava Am $3^{\text {rd }}$, et al. Anal ultrasound and endosonographic measurement of perineal body thickness: A new evaluation for fecal incontinence in females. Surg Endosc. 2004; 18(4): 650-4.

14. Starck M, Bohe M, Valentin L. Results of endosonographic imaging of the anal sphincter 2-7 days after primary repair of third- or fourth-degree obstetric sphincter tears. Ultrasound Obstet Gynecol. 2003;22:609-15.

15. Siddiqui MR, Ashrafian H, Tozer P, Daulatzai N, Burling D, Hart A, Athanasiou T, Phillips RK. A diagnostic accuracy meta-analysis of endoanal ultrasound and MRI for perianal fistula assessment. Dis Colon Rectum. 2012;55:576-85.

16. Hildebrandt U, Feifel G. Preoperative staging of rectal cancer by intrarectal ultrasound. Dis Colon Rectum. 1985;28:42-6.

17. Doornebosch PG, Bronkhorst PJ, Hop WC, Bode WA, Sing AKde GraafEJ. The role of endorectalultrasound in therapeutic decision-making for local versus. Transabdominal resection of rectal cancer. Dis Colon Rectum. 2008;51(1):38-42.

18. Garcia-Granero E, Faiz O, Flor B, Garcia S, Esclapez, P, Cervantes A. Prognostic implications of circunferential location of distal rectal cancer. Colorrectal Dis. 2011;13(6):650-7.

19. Giovannini M, Bardou VJ, Barclay R, Palazzo L, Roseau G, Helbert T, et al. Anal carcinoma: Prognostic value of endorectal ultrasound. Results of a prospective multicenter study. Endoscopy. 2001:33(3):231-6.

20. Parikh J, Shaw A, Grant LA, Schizas AM, Datta V, Williams AB, Griffin N. Anal carcinomas: The role of endoanal ultrasound and magnetic resonance imaging in staging, response evaluation and follow-up. Eur Radiol. 2011;21:776-85.

21. Martellucci J. Endoanal ultrasound for anal cancer follow up. Int J Colorectal Dis. 2011;26:679-80.

22. Beer-Gabel M, Carter D, Venturero M, Zmora O, Zbar AP. Ultrasonographic assessment of patients referred with chronic anal pain to a tertiary referral centre. Tech Coloproctol. 2010;14(2):107-12.

23. Naldini G, Cerullo G, Mascagni D, Orlandi S, Menconi C, Zeri K, Felli E, Martellucci J. Hiding intersphincteric and transphincteric sepsis in a novel pathological approach to chronic anal fissure. Surg Innov. 2012;19:33-6.

24. Stamatiadis A. Endoanal ultrasound in perianal abscesses. Dis Colon Rectum. 2002;45:710-1.

25. Millan M, García-Granero E, Esclápez P, Flor-Lorente B, Espí A, Lledó S. Management of intersphincteric abscesses. Colorectal Dis. 2006;8:777-80.

26. AG, Parks, PH Gordon, JD Hardcastle. A classification of fistula-in-ano. BR J Surg. 1976;63:1-12.

27. Cho Dong-Yoon. Endosonographic criteria for an internal opening of fistula-in ano. Dis Colon Rectum. 1999;42 (4):515-8. 